\title{
Shear-wave elastography: a new potential method to diagnose ulnar neuropathy at the elbow
}

\author{
Łukasz Paluch $^{1} \cdot$ Bartłomiej Noszczyk ${ }^{2} \cdot$ Żaneta Nitek $^{1} \cdot$ Jerzy Walecki ${ }^{1} \cdot$ Katarzyna Osiak $^{2} \cdot$ Piotr Pietruski $^{2,3}$
}

Received: 7 January 2018 / Revised: 19 April 2018 / Accepted: 26 April 2018 / Published online: 1 June 2018

(C) The Author(s) 2018

\begin{abstract}
Key Points

- SWE enables reliable detection of cubital tunnel syndrome

- Significant increase of entrapped ulnar nerve stiffness is observed in UNE

- SWE is a perspective screening tool for early detection of compressive neuropathies
\end{abstract}

Objectives The primary aim of this study was to verify if shear-wave elastography (SWE) can be used to diagnose ulnar neuropathy at the elbow (UNE). The secondary objective was to compare the cross-sectional areas (CSA) of the ulnar nerve in the cubital tunnel and to determine a cut-off value for this parameter accurately identifying persons with UNE.

Methods The study included 34 patients with UNE (mean age, 59.35 years) and 38 healthy controls (mean age, 57.42 years). Each participant was subjected to SWE of the ulnar nerve at three levels: in the cubital tunnel (CT) and at the distal arm (DA) and mid-arm (MA). The CSA of the ulnar nerve in the cubital tunnel was estimated by means of ultrasonographic imaging.

Results Patients with UNE presented with significantly greater ulnar nerve stiffness in the cubital tunnel than the controls (mean, $96.38 \mathrm{kPa}$ vs. $33.08 \mathrm{kPa}, p<0.001$ ). Ulnar nerve stiffness of $61 \mathrm{kPa}, \mathrm{CT}$ to DA stiffness ratio equal 1.68, and CT to MA stiffness ratio of 1.75 provided $100 \%$ specificity, sensitivity, positive and negative predictive value in the detection of UNE. Mean CSA of the ulnar nerve in the cubital tunnel turned out to be significantly larger in patients with UNE than in healthy controls $(p<0.001)$. A weak positive correlation was found in the UNE group between the ulnar nerve CSA and stiffness $(\mathrm{R}=0.31, p=0.008)$.

Conclusions SWE seems to be a promising, reliable and simple quantitative adjunct test to support the diagnosis of UNE.

Keywords Elastography $\cdot$ Cubital tunnel syndrome $\cdot$ Ulnar nerve $\cdot$ Ulnar nerve entrapment syndrome $\cdot$ Ulnar neuropathies

\author{
Abbreviations \\ CSA Cross-sectional areas \\ CT Cubital tunnel \\ DA Distal arm \\ EMG Electromyography \\ FCU Flexor carpi ulnaris
}

$\begin{array}{ll}\text { MA } & \text { Mid-arm } \\ \text { MRI } & \text { Magnetic resonance imaging } \\ \text { ROI } & \text { Region of interest } \\ \text { SWE } & \text { Shear-wave elastography } \\ \text { UNE } & \text { Ulnar neuropathy at the elbow } \\ \text { US } & \text { Ultrasonography }\end{array}$

\section{Introduction}

Cubital tunnel syndrome, also referred to as ulnar neuropathy at the elbow (UNE), is the second most common peripheral entrapment neuropathy after carpal tunnel syndrome. It can be defined as a compression of the ulnar nerve at the level of the elbow or in its direct proximity [1-4]. The ulnar nerve may be compressed at the cubital tunnel inlet by the medial intermuscular septum and an aponeurotic band referred to as the arcade of Struthers. Moreover, the nerve may be entrapped 
in the tunnel by the Osborne's band, a retinaculum that extends from the medial epicondyle to the olecranon, and by the aponeurosis of the flexor carpi ulnaris (FCU); this is postulated to be the most common site of the entrapment [5-7]. Finally, the ulnar nerve may be compressed distally, at the tunnel outlet, by the two heads of the FCU muscle [7].

Idiopathic neuropathy seems to be the most common cause of UNE among many potential aetiological factors of this condition $[7,8]$. Ulnar neuropathy may also result from habitual elbow flexion, acute or repetitive trauma, excessive strain, presence of mass-like lesions, degenerative arthritis and snapping triceps syndrome. The list of intrinsic aetiological factors includes diseases of the thyroid, diabetes mellitus, alcohol abuse, rheumatoid arthritis and other systemic inflammatory diseases, to mention a few $[5,7,9-11]$.

Because of its high incidence in the general population and a broad spectrum of symptoms that may interfere with patients' ability to work and their activities of daily living, UNE constitutes a significant clinical problem $[7,8,12]$. A number of healthcare professionals with various specialities, among them general practitioners, plastic surgeons, orthopaedic surgeons and neurologists, are involved in the evaluation of UNE. Although no consensus regarding a management algorithm for UNE has been reached thus far, a recent metaanalysis conducted by Mowlavi et al. demonstrated that clinical work-up is crucial to establish the diagnosis, to determine the severity of ulnar neuropathy and to choose an appropriate treatment strategy [12]. The diagnosis is based primarily on detailed analysis of medical history and physical examination. According to many authors, electrodiagnostic studies constitute an essential component of the evaluation, as they can be used to confirm the diagnosis and to assess the severity of nerve damage. Additional tests, such as ultrasonography (US), plain film radiography and magnetic resonance imaging (MRI), are recommended in patients with vague clinical presentation or during preoperative work-up [1, 5-7, 13-25]. Based on published evidence we can expect that also diffusion tensor imaging may be soon added to the peripheral nerve assessment armamentarium [26-31].
During recent years, sonoelastography, a relatively new imaging technology to quantify tissue stiffness, has been gaining a growing interest of researchers and healthcare professionals. Since its introduction by Ophir and colleagues in 1991, sonoelastography has evolved considerably and found application in many clinical disciplines; other potential uses of sonoelastography are still a subject of ongoing research [32, 33]. One highly promising application of sonoelastography, in particular shear-wave elastography (SWE), is the evaluation of peripheral nerve elasticity in patients with entrapment syndromes. Several authors reported the use of elastography in the diagnosis of median, sciatic and tibial compressive neuropathies [34-43]. However, to the best of our knowledge, potential application of this technique as a method to detect UNE has not been studied thus far.

The aim of this study was to use SWE for the quantification of ulnar nerve elasticity in patients with UNE and in healthy controls, and to verify if this technique may find application in clinical practice as an instrument to detect compressive neuropathy.

\section{Materials and methods}

\section{Study protocol}

Protocol of the study was approved by the local bioethics committee, and written informed consent was sought from all participants. A plastic surgeon assigned the study subjects to one of two groups, based on the inclusion criteria presented in Table 1.

Each study participant was subjected to US and SWE, both conducted with a Toshiba iAplio 900 ultrasonograph equipped with a 5-18 MHz transducer (Canon Medical Systems). Both US and SWE were carried out during the same visit, by the same radiologist with more than 4 years of experience in both diagnostic methods, blinded to subjects' clinical history, physical and results of electrodiagnostic testing (EDX). The examination was performed in a seated position, with the subject facing the examiner. The examined arm was kept downwards
Table 1 Inclusion criteria of the study

\begin{tabular}{ll}
\hline UNE group & Control group \\
\hline UNE symptoms present on physical examination & No evidence of UNE symptoms on physical examination \\
UNE confirmed by EDX & UNE excluded by EDX \\
No history of elbow surgery & \\
No history of humeral or ulnar fracture & \\
No systemic neurological disorder & \\
No thyroid disorder & \\
No diabetes mellitus & \\
No current pregnancy
\end{tabular}

$E D X$, Electrodiagnostic testing; UNE, Ulnar neuropathy at the elbow 
alongside the trunk, with flexed elbow and forearm resting on the knee in a supine position. To control and minimise the pressure applied onto skin surface, an even layer of hydrogel was applied onto the examined area.

During B-mode US examination of the cubital tunnel, the transducer was maintained perpendicularly to the ulnar nerve. Transverse cross-sections of the ulnar nerve were acquired at medial epicondyle level to determine the cross-sectional area of the nerve (CSA, in $\mathrm{mm}^{2}$ ). To measure the CSA, the ulnar nerve margin was traced along the inner border of perineural echogenic rim, corresponding to the perineurium around the hypoechoic nerve.

Ulnar nerve stiffness was measured at three levels labelled with a skin marker prior to the examination: in the cubital tunnel at the level of medial epicondyle and at a posteromedial aspect of the arm, $5 \mathrm{~cm}$ and $10 \mathrm{~cm}$ proximally to the cubital tunnel inlet (i.e. on the distal and mid-arm, respectively) (Fig. 1). The course of the ulnar nerve at each level was localised on B-mode scans. Then, the transducer was rotated 90 degrees to obtain SWEmode images of the nerve in a longitudinal axis. The transducer was always kept parallel to skin surface, and special attention was paid to minimise the pressure and to avoid a disruption of the hydrogel layer. Nerve stiffness at each level was measured three times with 2-3 min intervals and mean measurement was used for further processing; during the examination, the ulnar

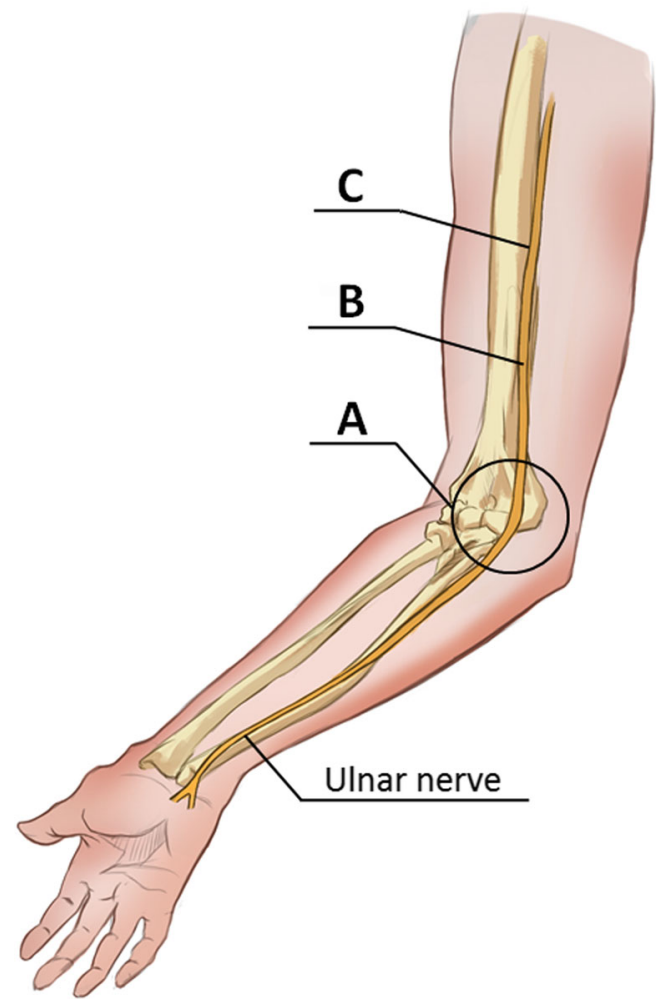

Fig. 1 Ulnar nerve segments subjected to elastographic examination. A, Cubital tunnel at medial epicondyle level; B, Posteromedial arm, $5 \mathrm{~cm}$ proximally to the cubital tunnel inlet (distal arm level); C, Posteromedial arm, $10 \mathrm{~cm}$ proximally to the cubital tunnel inlet (mid-arm level) nerve was always positioned in the centre of the $1 \mathrm{x} 1 \mathrm{~cm}$ Q-box, set in such way that no underlying bone surfaces were captured on longitudinal ultrasonographic scans. Shear modulus data for the selected circular region of interest (ROI, $2 \mathrm{~mm}$ in diameter) were acquired automatically by the ultrasonographic software; the results were expressed in kilopascals $(\mathrm{kPa})$. Representative elastographic images are presented in Fig. 2.

\section{Statistical analysis}

Normal distribution of the study variables was verified with Shapiro-Wilk test. Statistical characteristics of continuous variables were presented as means, medians and ranges. The characteristics of qualitative variables were shown as numbers and percentages. The significance of intergroup differences in the statistical characteristics of continuous variables was verified with Mann-Whitney U-test and Kruskal-Wallis test with Dunn post-hoc tests, whereas the significant of intragroup differences was tested with Wilcoxon signed-rank test. Diagnostic accuracy of ulnar nerve stiffness and CSA in the detection of UNE was verified by means of receiver operating characteristic (ROC) analysis. Sensitivity, specificity, positive and negative predictive value (PPV and NPV, respectively) of each potential predictor of UNE were calculated, along with the area under the ROC curve (AUC) and its 95\% confidence interval $(95 \% \mathrm{CI})$. All calculations were carried out with Statistica 10 package (StatSoft), with the threshold of statistical significance set at $p \leq 0.05$.

\section{Results}

UNE group included 34 patients with mean age of 59.35 years (range, 36-85 years), among them six men (mean age, 62 years; range, $48-85$ years) and 28 women (mean age, 58,79 years; range, 36-80 years), with confirmed ulnar compressive neuropathy at the elbow level. Control group comprised 38 healthy volunteers with mean age of 57.42 years (range, 38-84 years), among them three men (mean age, 53.67 years; range, 38-75 years) and 35 women (mean age, 57.74 years; range, 38-84 years), in whom UNE was excluded based on the results of physical examination and EDX.

The CSA of the ulnar nerve at the cubital tunnel level turned out to be significantly larger in patients with UNE than in the controls (mean, $9.03 \mathrm{~mm}^{2}$; median, $8 \mathrm{~mm}^{2}$; range, $5-18$ $\mathrm{mm}^{2}$ vs. mean, $6.47 \mathrm{~mm}^{2}$; median, $6 \mathrm{~mm}^{2}$; range, $3-9 \mathrm{~mm}^{2} ; p$ $<0.001)$. The cut-off value for the CSA of the ulnar nerve at the cubital tunnel level, which most accurately distinguished between the individuals with the entrapment neuropathy and without was $10 \mathrm{~mm}^{2}$; ROC analysis demonstrated that this value provided $38.2 \%$ sensitivity, $100 \%$ specificity, $100 \%$ PPV and $64.4 \%$ NPV in the detection of UNE (Fig. 3). 

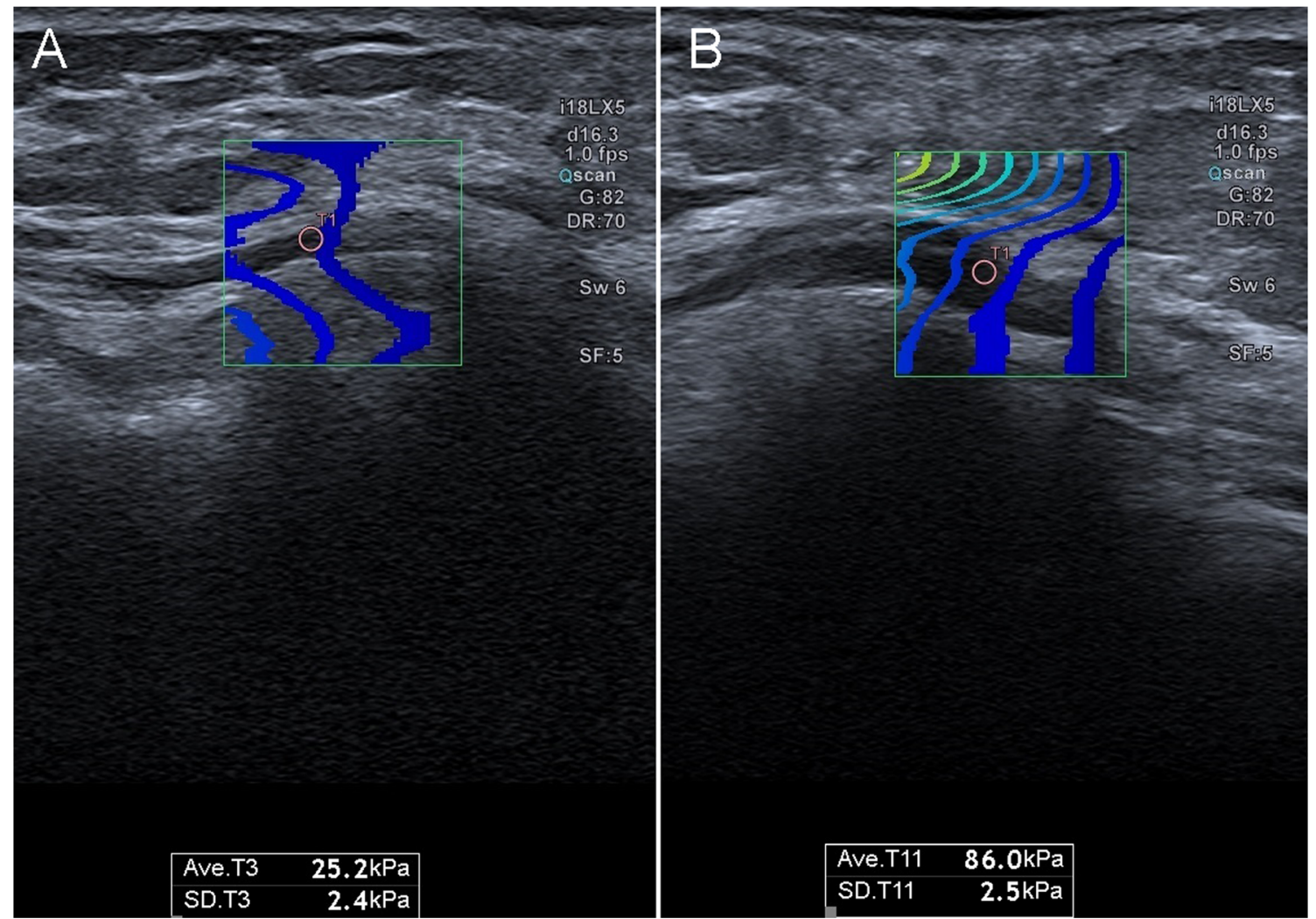

Fig. 2 Elastographic presentation of ulnar nerve at the elbow level in (A) healthy individual (low SWE value), and (B) in patient with compressive neuropathy (high SWE value)

The results of SWE measurements are summarised in Table 2 . In any patient the differences between measurements that were repeated at each level with 3 minutes intervals did not exceed $2 \mathrm{kPa}$ ( $\mathrm{SD} \geq 1.15$ ). Compared to the controls, patients with UNE presented with significantly greater ulnar nerve stiffness at each examined level. In the UNE group, mean ulnar nerve stiffness at the entrapment site in the cubital tunnel $(96.38 \mathrm{kPa})$ was significantly greater than at the distal arm and mid-arm $(38.79 \mathrm{kPa}$ and $39.06 \mathrm{kPa}$, respectively; $p<$ 0.001 ). In the healthy controls, no statistically significant differences were found in ulnar nerve stiffness at various levels $(p=0.779)$.

Aside from the comparison of crude SWE parameters for the entrapment site, we also analysed their normalised values, obtained by dividing ulnar nerve stiffness at the cubital tunnel level (CT) by its stiffness at the distal arm (CT to DA ratio) or mid-arm (CT to MA ratio). The values of both ratios turned out to be significantly higher in patients with UNE than in the controls $(p<0.001)$ (Table 3).

ROC curves illustrating the diagnostic accuracy of elastographically determined ulnar nerve stiffness at the cubital tunnel level, CT to DA and CT to MA ratios in the detection of UNE are presented in Fig. 3. Ulnar nerve stiffness at the cubital tunnel level equal $61 \mathrm{kPa}$ provided $100 \%$ specificity, sensitivity, PPV and NPV in the detection of UNE. Equally excellent indices of diagnostic accuracy were also obtained for the $\mathrm{CT}$ to DA ratio of 1.68 , and $\mathrm{CT}$ to MA ratio of 1.75 (Fig. 3).

A weak positive correlation was found in the UNE group between the CSA of the ulnar nerve and its stiffness at the site of compression ( $\mathrm{R}=0.31, p=0.008)$. Moreover, the CSA of the ulnar nerve correlated significantly with the values of CT to $\mathrm{DA}(\mathrm{R}=0.31, p=0.007)$ and $\mathrm{CT}$ to MA ratios $(\mathrm{R}=0.30, p$ $=0.010$ ).

\section{Discussion}

We hypothesised that SWE can be used for an objective evaluation of oedema and fibrosis associated with ulnar nerve compression. The study showed clearly that patients with UNE presented with significantly greater ulnar nerve stiffness at the cubital tunnel level than healthy controls (mean, $96.38 \mathrm{kPa}$ vs. $33.08 \mathrm{kPa}$ ). Furthermore, stiffness of the ulnar nerve in UNE patients turned out to be significantly greater at the entrapment site than at the mid- and distal arm; however, a similar relationship was not observed in the controls. ROC analysis demonstrated that UNE could be accurately diagnosed whenever stiffness of the ulnar nerve at the entrapment site equalled $61 \mathrm{kPa}$ or more. However, recall that ulnar nerve stiffness may vary from patient to patient due to the modulatory effect of additional factors, such as sex, age, BMI, 

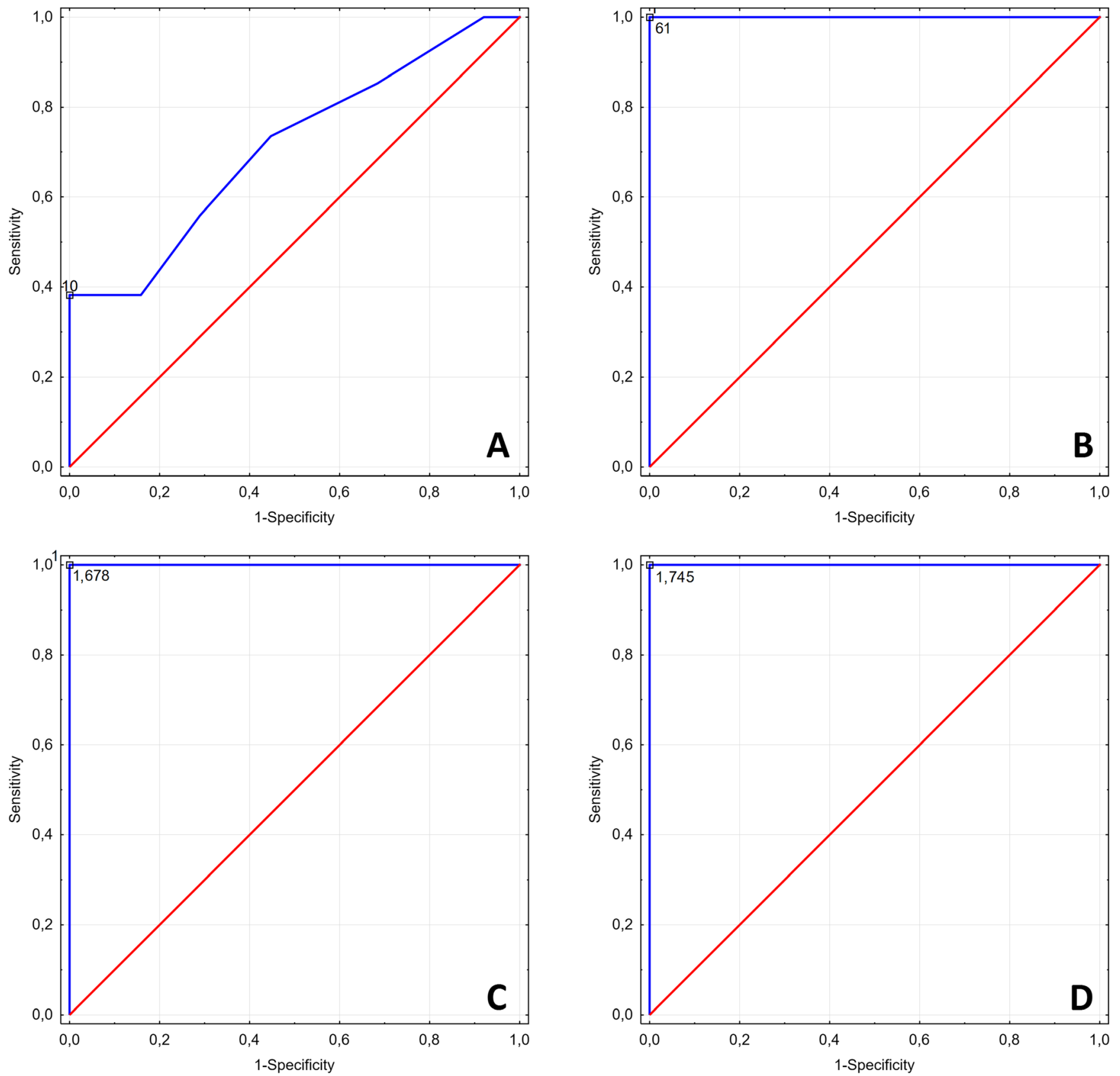

Fig. 3 ROC curve illustrating diagnostic accuracy of (A) the cross-sectional area of ulnar nerve $\left(10 \mathrm{~mm}^{2}\right),(\mathbf{B})$ ulnar nerve stiffness at the entrapment site (61 kPa), (C) CT to DA ratio (1.68), and (D) CT to MA ratio (1.75) in the detection of UNE

occupation, and upper limb physiognomy. Thus, to boost the diagnostic accuracy of SWE, instead of using the crude value of ulnar nerve stiffness at the entrapment site, we recommend a normalised value of this parameter; dividing ulnar nerve stiffness at the entrapment site by the stiffness of its intact segment, one can eliminate individual variability in the elastographic parameters. In this study, we divided ulnar nerve stiffness at the entrapment site by its stiffness at the distal and mid-arm, obtaining CT to DA and CT to MA ratios, respectively. Similar to the crude value of ulnar nerve stiffness, also the values of both ratios were significantly higher in patients with CTS than in the controls. Moreover, ROC analysis demonstrated that the cut-off values for CT to DA and CT to MA ratios (1.68 and 1.75 , respectively) accurately distinguished between patients with compressive ulnar neuropathy and without.

As mentioned before, the diagnosis of UNE is based primarily on medical history and physical examination. Although this diagnosis is usually confirmed by EDX, US due to recent implementation of high-frequency transducers enabling high-resolution images of peripheral nerves is emerging as a complementary method to evaluate patients 
Table 2 Elastographic estimates of ulnar nerve stiffness (in $\mathrm{kPa}$ ) in healthy volunteers (control group) and patients with confirmed cubital tunnel syndrome (UNE group)

\begin{tabular}{|c|c|c|c|c|c|c|c|c|c|}
\hline \multirow[t]{2}{*}{ Ulnar nerve SWE value } & \multicolumn{4}{|c|}{ Control group $(n=38)$} & \multicolumn{4}{|c|}{ UNE group (n=34) } & \multirow[t]{2}{*}{$p$} \\
\hline & Mean & Median & Range & SD & Mean & Median & Range & SD & \\
\hline Cubital tunnel level & 33.08 & 31 & $19-51$ & 10.13 & 96.38 & 98 & $61-121$ & 9.62 & $<0.001 *$ \\
\hline Distal arm level & 33.32 & 33 & $21-51$ & 9.13 & 38.79 & 36,5 & $21-65$ & 11.33 & 0.041 \\
\hline Mid-arm level & 33.18 & 31 & $19-52$ & 8.82 & 39.06 & 36 & $20-61$ & 10.45 & 0.009 \\
\hline
\end{tabular}

*Statistically significant intergroup difference

with compressive neuropathies, e.g. UNE [5, 7, 15, 17, 22-25, 44, 45]. The hallmark sonographic features of entrapment neuropathy include enlargement of the nerve, its hypoechoic swelling proximal to the compression site, loss of normal fascicular pattern and, not infrequently, oedema of adjacent soft tissues $[7,13,20,22,23]$. US is also suitable for the detection of space-occupying masses. Importantly, during the examination, ultrasonographic images of the affected limb can be compared with those of the contralateral extremity. Highresolution US was shown to be more cost- and timeeffective than MRI, and its availability is markedly higher. Noticeably, US can be used for both static and dynamic assessment of the ulnar nerve; this may be particularly helpful in patients with nerve subluxation or snapping triceps syndrome $[5,7]$. Previous studies demonstrated that ultrasonographically determined ulnar nerve CSA was a feasible indicator for UNE $[5,25,46]$; ulnar nerve CSA at the elbow level equal to $8.3 \mathrm{~mm}^{2}$ provided high specificity and sensitivity in the detection of ulnar nerve entrapment $[5,17$, $24,25]$. Our findings seem to be consistent with these observations. Not only did the mean CSA of the ulnar nerve at the cubital tunnel level turn out to be significantly larger in patients with UNE than in healthy controls (9.03, range, 5-18 $\mathrm{mm}^{2}$ vs. 6.47 , range, $3-9 \mathrm{~mm}^{2}$ ), but also a significant positive correlation was found between this parameter and the elastographically determined ulnar nerve stiffness at the entrapment site. The latter observation seems to confirm the applicability of SWE to UNE diagnosis. The principal drawback of peripheral nerve ultrasonography is the lack of a standardised examination protocol (examination technique, type of transducer, wave frequency) [7]. Moreover, the examination is to a large degree operator-dependent, and thus, its result can be considered reliable only if conducted by an experienced examiner.

Our findings are consistent with the results of other studies that analysed potential application of elastography in the evaluation of compression neuropathies; also, those studies demonstrated that compression may contribute to greater stiffness of the entrapped nerve [34-43]. In our opinion, SWE constitutes a valuable supplement to ultrasonographic examination of peripheral nerves, also in patients with UNE [14]. Elastography is both a time- and cost-effective method with a relatively steep learning curve. Elastographic findings add considerably to the results of US, providing quantitative data on the nerve stiffness during both static and dynamic examination. In addition, it can determine the level of nerve entrapment. In the future, with its growing availability in routine clinical practice, SWE may be used for the monitoring of treatment outcomes in UNE patients. The numerous advantages of SWE make it a perspective screening tool for early detection of compressive neuropathies, with potential application in occupational medicine. Prompt introduction of adequate preventive measures in persons at risk or implementation of conservative treatment in individuals with early stages of the neuropathy may prevent its long-term consequences, such as chronic disability.

The main limitation of this study may stem from the fact that ulnar nerve stiffness was determined by one radiologist, and thus, we were unable to estimate inter-observer reliability of the results. During the course of another study, which is soon to be conducted at our centre, we will analyse both interand intra-observer reliability of SWE measurements taken at various levels of ulnar and median nerves. Interestingly, patients from UNE group presented with significantly greater
Table 3 Normalised values of ulnar nerve stiffness (ratios) in healthy volunteers (control group) and patients with confirmed cubital tunnel syndrome (UNE group)

\begin{tabular}{lccccccc}
\hline Ulnar nerve SWE ratio & \multicolumn{2}{c}{ Control group $(\mathrm{n}=38)$} & & \multicolumn{2}{l}{ UNE group $(\mathrm{n}=34)$} & & $p$ \\
\cline { 2 - 3 } & Median & Range & & Median & Range & \\
\hline Cubital tunnel level : Distal arm level & 1.0 & $0.8-1.2$ & & 2.8 & $1.7-4.0$ & $<0.001 *$ \\
Cubital tunnel level : Mid-arm level & 1.0 & $0.7-1.2$ & & 2.7 & $1.7-4.1$ & $<0.001 *$ \\
\hline
\end{tabular}

*Statistically significant intergroup difference 
ulnar nerve stiffness proximally to the compression site than the controls. Because of the preliminary characteristic of our study and lack of evidence from similar study, we are unable to provide an unequivocal explanation for this phenomenon. The exact pathophysiology of compressive neuropathy is still not fully understood. It is commonly accepted that increased pressure on a peripheral nerve affects its microcirculation and, therefore, blood supply. Resultant epineural ischaemia contributes to impaired venous outflow and stasis. Chronic compression may predispose to capillary leakage and neural oedema, which further aggravates the harmful effects of the primary causal factor. With time, this mechanism induces inflammation, fibrosis, demyelination and eventually, after a remyelination failure, axonal integrity loss [7, 47-49]. Perhaps, this process involves the segment located proximally to the compression site to a larger degree than previously supposed, which manifests as greater stiffness of the nerve, likewise in our series. This would suggest that elastography may be a highly sensitive test to detect structural anomalies in peripheral nerves. This issue definitively needs to be addressed in future studies.

The results of this preliminary study justify further research on the application of SWE as a diagnostic instrument in ulnar neuropathies. The future studies should involve larger groups of patients and verify whether SWE is suitable for the staging of ulnar nerve dysfunction and if the elastographic indices correlate with the routinely used EDX-based scale of UNE severity. Moreover, future research should corroborate if elastography could be used as a screening tool for peripheral neuropathies and whether it may find application as a method to monitor the treatment outcomes.

\section{Conclusions}

To summarise, this study demonstrated for the first time that shear wave elastography can be used to diagnose cubital tunnel syndrome. Patients with entrapment syndrome presented with significantly greater ulnar nerve stiffness at the elbow level than healthy controls. These findings imply that SWE has a potential to become a routine diagnostic instrument to detect ulnar neuropathy in a clinical setting.

Funding The authors state that this work has not received any funding.

\section{Compliance with ethical standards}

Guarantor The scientific guarantor of this publication is Bartłomiej Noszczyk.

Conflict of interest The authors of this manuscript declare no relationships with any companies, whose products or services may be related to the subject matter of the article.
Statistics and biometry One of the authors has significant statistical expertise.

Informed consent Written informed consent was obtained from all subjects (patients) in this study.

Ethical approval Institutional Review Board (The Bioethical Committee of the Medical Centre of Postgraduate Education in Warsaw) approval was obtained.

\section{Methodology}

- prospective

- diagnostic or prognostic study

- multicentre study

Open Access This article is distributed under the terms of the Creative Commons Attribution 4.0 International License (http:// creativecommons.org/licenses/by/4.0/), which permits unrestricted use, distribution, and reproduction in any medium, provided you give appropriate credit to the original author(s) and the source, provide a link to the Creative Commons license, and indicate if changes were made.

\section{References}

1. Greenwald D, Blum LC III, Adams D et al (2006) Effective surgical treatment of cubital tunnel syndrome based on provocative clinical testing without electrodiagnostics. Plast Reconstr Surg 117:87-91

2. Assmus H, Antoniadis G, Bischoff C et al (2011) Cubital tunnel syndrome-a review and management guidelines. Cent Eur Neurosurg 72:90-98

3. Bartels RH, Menovsky T, Van Overbeeke JJ et al (1998) Surgical management of ulnar nerve compression at the elbow: an analysis of the literature. J Neurosurg 89:722-727

4. Mondelli M, Giannini F, Ballerini M et al (2005) Incidence of ulnar neuropathy at the elbow in the province of Siena (Italy). J Neurol Sci 234:5-10

5. Shen L, Masih S, Patel DB et al (2016) MR anatomy and pathology of the ulnar nerve involving the cubital tunnel and Guyon's canal. Clin Imaging 40:263-274

6. Boone S, Gelberman RH, Calfee RP (2015) The management of cubital Tunnel Syndrome. J Hand Surg Am 40:1897-1904

7. Tang DT, Barbour JR, Davidge KM et al (2015) Nerve entrapment: update. Plast Reconstr Surg 135:199-215

8. Carter GT, Weiss MD, Friedman AS et al (2015) Diagnosis and treatment of work-related unlar neuropathy at the elbow. Phys Med Rehabil Clin N Am 26:513-522

9. Harding IJ, Morris IM (2003) The aetiology and outcome of 170 ulnar nerve lesions confirmed with electrophysiological testing. Acta Orthop Belg 69:405-411

10. Spinner RJ, Goldner RD (1998) Snapping of the medial head of the triceps and recurrent dislocation of the ulnar nerve. Anatomical and dynamic factors. J Bone Joint Surg Am 80:239-247

11. Lasecki M, Olchowy C, Pawlus A et al (2014) The snapping Elbow Syndrome as a reason for chronic elbow neuralgia in a tennis player - MR, US and sonoelastography evaluation. Pol J Radiol 79:467471

12. Mowlavi A, Andrews K, Lille S et al (2000) The management of cubital tunnel syndrome: a meta-analysis of clinical studies. Plast Reconstr Surg 106:327-334

13. Omejec G, Žgur T, Podnar S (2015) Diagnostic accuracy of ultrasonographic and nerve conduction studies in ulnar neuropathy at the elbow. Clin Neurophysiol 126:1797-1804 
14. Beekman R, Visser LH, Verhagen WI (2011) Ultrasonography in ulnar neuropathy at the elbow: a critical review. Muscle Nerve 43: $627-635$

15. van Veen KE, Wesstein M, van Kasteel V (2014) Ultrasonography and electrodiagnostic studies in ulnar neuropathy: an examination of the sensitivity and specificity and the correlations between both diagnostic tools. J Clin Neurophysiol 32:240-243

16. Volpe A, Rossato G, Bottanelli M et al (2009) Ultrasound evaluation of ulnar neuropathy at the elbow: correlation with electrophysiological studies. Rheumatology (Oxford) 48:1098-1101

17. Kim JH, Won SJ, Rhee WI et al (2015) Diagnostic cutoff value for ultrasonography in the ulnar neuropathy at the elbow. Ann Rehabil Med 39:170-175

18. Kim S, Choi JY, Huh YM et al (2007) Role of magnetic resonance imaging in entrapment and compressive neuropathy: what, where, and how to see the peripheral nerves on the musculoskeletalmagnetic resonance image. Part 1. Overview and lower extremity. Eur Radiol 17: 139-149

19. Kim S, Choi JY, Huh YM et al (2007) Role of magnetic resonance imaging in entrapment and compressive neuropathy: what, where, and how to see the peripheral nerves on the musculoskeletal magnetic resonance image. Part 2. Upper extremity. Eur Radiol 17:509-522

20. Jacobson JA, Fessell DP, Lobo Lda G et al (2010) Entrapment neuropathies I: upper limb (carpal tunnel excluded). Semin Musculoskelet Radiol 14:473-486

21. Andreisek G, Crook DW, Burg D et al (2006) Peripheral neuropathies of the median, radial, and ulnar nerves: MR imaging features. Radiographics 26:1267-1287

22. Koenig RW, Pedro MT, Heinen CP et al (2009) High-resolution ultrasonography in evaluating peripheral nerve entrapment and trauma. Neurosurg Focus 26:1-6

23. Martinoli C, Bianchi S, Pugliese F et al (2004) Sonography of entrapment neuropathies in the upper limb (wrist excluded). $\mathrm{J}$ Clin Ultrasound 32:438-450

24. Choi S-J, Ahn JH, Ryu DS et al (2015) Ultrasonography for nerve compression syndromes of the upper extremity. Ultrasonography 34:275-291

25. Chang KV, Wu WT, Han DS et al (2017) Ulnar nerve crosssectional area for the diagnosis of cubital Tunnel Syndrome: a meta-analysis of ultrasonographic measurements. Arch Phys Med Rehabil. https://doi.org/10.1016/j.apmr.2017.08.467

26. Breitenseher JB, Kranz G, Hold A et al (2015) MR neurography of ulnar nerve entrapment at the cubital tunnel: a diffusion tensor imaging study. Eur Radiol 25:1911-1918

27. Bao H, Wang S, Wang G et al (2017) Diffusion-weighted MR neurography of median and ulnar nerves in the wrist and palm. Eur Radiol 27:2359-2366

28. Wu C, Wang G, Zhao Y et al (2017) Assessment of tibial and common peroneal nerves in diabetic peripheral neuropathy by diffusion tensor imaging: a case control study. Eur Radiol 27:35233531

29. Kronlage M, Schwehr V, Schwarz D et al (2017) Peripheral nerve diffusion tensor imaging (DTI): normal values and demographic determinants in a cohort of 60 healthy individuals. Eur Radiol. https://doi.org/10.1007/s00330-017-5134-z

30. Chhabra A, Madhuranthakam AJ, Andreisek G (2018) Magnetic resonance neurography: current perspectives and literature review. Eur Radiol 28:698-707

31. Klauser AS, Abd Ellah M, Kremser C et al (2018) Carpal tunnel syndrome assessment with diffusion tensor imaging: value of fractional anisotropy and apparent diffusion coefficient. Eur Radiol 28:1111-1117

32. Ophir J, Cespedes I, Ponnekanti H et al (1991) Elastography: aquantitative method for imaging the elasticity of biological tissues. Ultrason Imaging 13:111-134

33. Wells PN, Liang HD (2011) Medical ultrasound: imaging of soft tissue strain and elasticity. J R Soc Interface 8:1521-1549

34. Orman G, Ozben S, Huseyinoglu N et al (2013) Ultrasound elastographic evaluation in the diagnosis of carpal tunnel syndrome: initial findings. Ultrasound Med Biol 39:1184-1189

35. Park GY, Kim SK, Park JH (2011) Median nerve injury after carpal tunel injection serially followed by ultrasonographic, sonoelastographic, and electrodiagnostic studies. Am J Phys Med Rehabil 90:336-341

36. Kantarci F, Ustabasioglu FE, Delil S et al (2014) Median nerve stiffness measurement by shear wave elastography: a potential sonographic method in the diagnosis of carpal tunnel syndrome. Eur Radiol 24:434-440

37. Xin H, Hu HY, Liu B et al (2017) Ultrasound elastographic evaluation of the median nerve in hemodialysis with carpal tunnelsyndrome. J Med Ultrason 44:123-131

38. Miyamoto H, Halpern EJ, Kastlunger M et al (2014) Carpal tunnel syndrome: diagnosis by means of median nerve elasticityimproved diagnostic accuracy of US with sonoelastography. Radiology 270:481-486

39. Martin MJ, Cartwright MS (2017) A pilot study of strain elastography in the diagnosis of carpal Tunnel Syndrome. J Clin Neurophysiol 34:114-118

40. Yoshii Y, Tung WL, Ishii T (2017) Strain and morphological changes of median nerve after carpal tunnel release. J Ultrasound Med 36: 1153-1159

41. Yoshii Y, Tung WL, Ishii T (2017) Measurement of median nerve strain and applied pressure for the diagnosis of carpal Tunnel Syndrome. Ultrasound Med Biol 43:1205-1209

42. Andrade RJ, Nordez A, Hug F et al (2016) Non-invasive assessment of sciatic nerve stiffness during human ankle motion using ultrasound shear wave elastography. J Biomech 49:326-331

43. Dikici AS, Ustabasioglu FE, Delil S et al (2017) Evaluation of the tibial nerve with shear-wave elastography: a potential sonographic method for the diagnosis of diabetic peripheral neuropathy. Radiology 282:494-501

44. Klauser AS, Abd Ellah MM, Halpern EJ et al (2015) Sonographic cross-sectional area measurement in carpal tunnel syndrome patients: can delta and ratio calculations predict severity compared to nerve conduction studies? Eur Radiol 25:2419-2427

45. Riegler G, Pivec C, Platzgummer H et al (2017) High-resolution ultrasound visualization of the recurrent motor branch of the median nerve: normal and first pathological findings. Eur Radiol 27:29412949

46. Wu CH, Chang KV, Ozcakar L et al (2015) Sonographic tracking of the upper limb peripheral nerves: a pictorial essay and video demonstration. Am J Phys Med Rehabil 94:740-747

47. Tapadia M, Mozaffar T, Gupta R (2010) Compressive neuropathies of the upper extremity: Update on pathophysiology, classification, and electrodiagnostic findings. J Hand Surg Am 35:668-677

48. Lundborg G, Dahlin LB (1996) Anatomy, function, and pathophysiology of peripheral nerves and nerve compression. Hand Clin 12: 185-193

49. Mackinnon SE (2002) Pathophysiology of nerve compression. Hand Clin 18:231-241 